\title{
Diagnostic value of acid phosphatases (ACP) in differentiating reactive mesothelial cells from cancer cells in the body fluid effusions
}

\author{
Yun-Hui Li ${ }^{1}$, Yang Wang ${ }^{2}$, Xiu-Xia Chen ${ }^{3}$, Bin Liang ${ }^{4}$, Xue-Shan Qiu ${ }^{5}$ En-Hua Wang ${ }^{5}$, Guang-Ping Wu ${ }^{5}$ \\ ${ }^{1}$ Department of Pathology, The First Affiliated Hospital and Department of Laboratory Medicine, No.202 Hospital, China Medical University, \\ Shenyang 110001, China; ${ }^{2}$ Department of Pathophysiology, College of Basic Medical Sciences, Dalian Medical University, Dalian 116044, China; \\ ${ }^{3}$ Department of Surgery, The First Hospital of China Medical University, Shenyang 110001, China; ${ }^{4}$ Department of Bioinformatics, College of Basic \\ Medical Sciences, China Medical University, Shenyang 110122, China; ${ }^{5}$ Department of Pathology, The First Affiliated Hospital and College of Basic \\ Medical Sciences, China Medical University, Shenyang 110001, China \\ Contributions: (I) Conception and design: YH Li, GP Wu; (II) Administrative support: XS Qiu, EH Wang, GP Wu; (III) Provision of study materials \\ or patients: YH Li, Y Wang, XX Chen, GP Wu; (IV) Collection and assembly of data: YH Li, XX Chen; (V) Data analysis and interpretation: YH Li, \\ B Liang; XS Qiu, EH Wang, GP Wu; (VI) Manuscript writing: All authors; (VII) Final approval of manuscript: All authors. \\ Correspondence to: Guang-Ping Wu, PhD. Department of Pathology, The First Affiliated Hospital and College of Basic Medical Sciences, China \\ Medical University, No.155 Nanjing Bei Street, Heping District, Shenyang 110001, China. Email: wug_ping@sina.com.
}

\begin{abstract}
Background: The cytological diagnosis of a malignant epithelial tumor, i.e., a cancer cell in the body fluid effusions is usually made by cytomorphological examination alone; however, diagnostic challenges can occur when the cancer cells are rare or cytological atypia is minimal. Morphological similarity between the cancer and the reactive mesothelial cell is the most common problem in establishing a clear diagnosis. The aim of this study is to investigate whether the cocktail acid phosphatases (ACP) special staining will be a useful tumor marker in differentiation of the reactive mesothelial cells from the cancer cells in the body fluid effusions.

Methods: The cocktail ACP special staining was performed on 212 body fluid effusion samples, which included 128 pleural effusions, 69 ascites, and 15 pericardial effusions.

Results: The mesothelial cells were cocktail ACP positive in 84 out of 84 benign effusion cases, and the sensitivity and the specificity were $100 \%$ for the benign effusions which including pleural effusions, ascites, and pericardial effusions. On the other hand, 122 out of 128 cancer cases were cocktail ACP negative, indicating that the sensitivity of using the cocktail ACP staining to rule out the malignant effusions was 95.3\%. Thus, the cocktail ACP staining is an excellent marker with high sensitivity and specificity to distinguish the carcinoma from the reactive mesothelial cells in the body fluid effusions.

Conclusions: Our finding provided a new tool for cytopathologists in diagnosing the body fluid effusion that could impact clinical decision making.
\end{abstract}

Keywords: Cytopathology; effusion; acid phosphatases (ACP); mesothelial cells, carcinoma

Submitted Mar 21, 2018. Accepted for publication Nov 05, 2018.

doi: $10.21037 /$ jtd.2018.11.64

View this article at: http://dx.doi.org/10.21037/jtd.2018.11.64

\section{Introduction}

The body fluid effusions include but are not restricted to pleural effusion, ascites, and pericardial effusion. They are one of the most common complications of benign and malignant diseases (1). The distinction of the benign from the malignant effusion depends primarily on cell morphology, and clinical treatment usually relies heavily on cytological diagnosis. The malignant effusion is mainly 
Table 1 The detail diagnostic information of the serous fluids for the adenocarcinoma patients

\begin{tabular}{lcc}
\hline Groups & Diagnosis & N \\
\hline Pleural & Lung cancer & 76 \\
& Breast cancer & 3 \\
Ascites & Gastric cancer & 11 \\
& Intestinal cancer & 9 \\
& Ovary cancer & 20 \\
Pericardial & Lung cancer & 9 \\
Total & - & 128 \\
\hline
\end{tabular}

caused by metastatic carcinomas, while the benign effusions can be caused by reactive mesothelial cells, macrophages and sometimes inflammatory cells. Because the morphologies between the carcinoma and the reactive mesothelial cells are quite similar, cytological differentiation of effusions based on morphology alone can be challenging, especially when the number of cancer cells is rare or the atypia is minimal (2). It has been reported recently that conventional cytological differential diagnosis had a sensitivity of $69.2 \%$ and a specificity of $95.0 \%$ for the detection of malignant cells in serous effusion samples (3). Over years, various techniques have been developed and applied to increase the diagnostic sensitivity and the specificity for the effusion samples, such as immunocytochemistry (ICC), electron microscopy, flow cytometry, image morphometry, FISH, PCR, and HLA antigen (4).

The ICC markers have been used as a powerful complementary tool to assist the cytological diagnosis of the malignant effusions (5-7). The majority of these markers are used to identify carcinoma cells, including Moc-31 (epithelial specific antigen/Ep-CAM), Ber-Ep4 (epithelial cell adhesion molecule), thyroid transcription factor 1 (TTF-1) and other emerging markers. Intracellular hyaluronic acid and CD44 had been used for differentiation mesothelioma from adenocarcinoma (8). Recently, more research on a family of enzymes, acid phosphatases (ACPs) have emerged. The members of this family enzyme have different molecular sizes and specific substrates. At least six different ACPs had been reported and used as tumor markers in human tissues (9-11). The erythrocytic (ACP1) and the lysosomal (ACP2) were broadly expressed by many types of cells. While the prostatic (ACP3), the macrophagic (ACP5), ACP6 and the testicular (ACPT) forms were expressed by more limited type of cells. Goto et al. found that the serum ACP level was increased in giant cell tumor of bone (GCT, benign) and claimed that the ACP could be used as a tumor marker for GCT (12). Markovic et al. reported that the ACP was useful as a tumor marker for screening cervical intraepithelial lesions on Pap smears (13). ACP was also used as a diagnostic marker for adult-onset Pompe disease when typical vacuolated fibers were absent (14). Janckila et al. showed that tartrateresistant ACP (TRACP) was an immunohistochemical marker for inflammatory macrophages, and found that circulating TRACP could be used as a biomarker for chronic inflammatory activity in certain chronic inflammatory diseases (15). The histological detection of an ACP was also exploited in the diagnose of hairy cell leukemia, Gaucher's disease, and osteoclastoma (16). Prostatic ACP (ACP3) was expressed in prostate cancer bone metastases and promoted osteoblast differentiation (17). However, ACP has not been reported as a marker for identification of the reactive mesothelial cells.

The purpose of this study was to investigate the diagnostic values of the cocktail ACP staining in the body fluid effusions, especially in differentiation of the reactive mesothelial cells from other cancer cells.

\section{Methods}

\section{Patients}

The study was approved by institutional ethics board of the First Affiliated Hospital of China Medical University (No. 2016-125). We have obtained internal board approval and/or patients informed consent for this study. The body fluid effusion samples of 212 patients were collected from the laboratory of cytopathology at the First Affiliated Hospital of China Medical University between Apr. 1, 2016 and Jan. 1, 2017. The samples contained 128 pleural effusions, 69 ascites, and 15 pericardial effusions. In the 212 effusion samples, 128 cases were defined as malignant with adenocarcinoma cells and 84 cases were benign with reactive mesothelial cells. The detail information of all patients with adenocarcinoma was listed in Table 1. Of the 128 patients with the malignant effusions, 50 were men (39.06\%) and 78 were women (60.94\%), with a mean age of 61.3 years from 21 to 89 . Of 84 patients with the benign effusions, 44 were men $(52.38 \%)$ and 40 were women (47.62\%), with a mean age of 59.26 years from 22 to 90 . The effusions were considered malignant if malignant cells were found in the cytological examinations or in the biopsy 


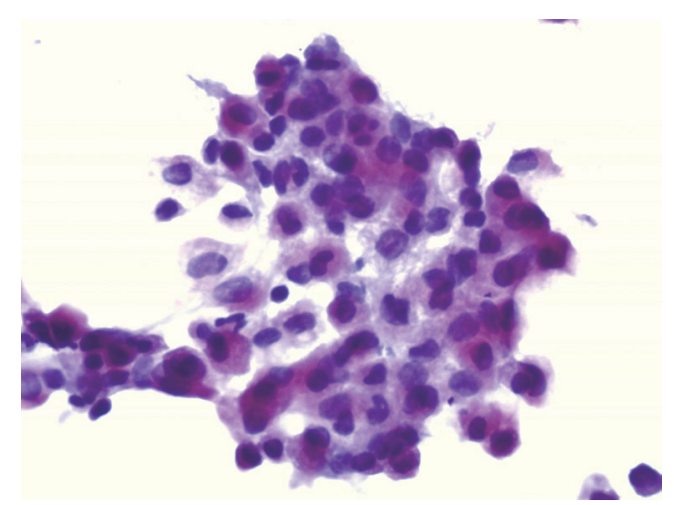

Figure 1 The cocktail ACP staining in an ascites patient. $80 \%$ of the reactive mesothelial cells were cocktail ACP staining positive: with strong pink color in the cytoplasm. Negative for the lymphocytes (no pink colors in the cytoplasm). ACP staining, $\times 400$. ACP, acid phosphatases.

specimens of pleural or peritoneum. Tuberculous and parapneumonic effusions were determined in the reference with PMID 22875282 (2).

\section{Sample preparation and ACP staining}

All effusion samples were freshly received, with a volume range of 20-2,000 $\mathrm{mL}$. The samples were centrifuged for 10 minutes at 2,000 g. Each resulting pellet was used for the preparation of two cytological smear slides, one for the routine cytological examination (alcohol fixed Papanicolaou stained) and the other used for the ACP staining. The cocktail ACP reagents were ordered from JCK Biopharmaceuticals, Hefei, China. It contained 5 kinds of ACPs and the main component was ACP5. The brief procedure for the cocktail ACP staining was: after the smears were fixed in $10 \%$ formalin for $4 \mathrm{~min}$ and washed in the buffer for $2 \mathrm{~min}$, the slides were then stained with the cocktail ACP staining reagent for 5 min strictly. After hematoxylin counter staining (JCK Biopharmaceuticals, Hefei) for $5 \mathrm{~min}$ and wash, the slides were mounted in a water-based Clear Mount mounting medium.

Two cytopathologists independently evaluated the staining blindly. When there were disagreements between the two cytologists, they reviewed the results together until the results were consistent. The data was expressed as frequencies which included the staining intensities and the percentages of the positive signals. The intensity was further divided into 3 levels (low, moderate, and high). The low intensity was defined as light pink cytoplasm which almost identical with the background. Thus, the low intensity was interpreted as negative. The moderate intensity was defined as a moderate (deep) pink cytoplasm which was significantly stronger than the background, and the high intensity was defined as a cytoplasm dyed marked (strongest) pink. The positive ACP staining was further defined that there were more than $5 \%$ of tumor cells or reactive mesothelial cells at least with a moderate intensity stain.

\section{Statistical analysis}

The sensitivity, the specificity, and the accuracy were calculated to evaluate the diagnostic performance of the ACP staining. A $P$ value of $<0.05$ was considered significant. The statistical analysis was performed using SPSS16.0 software (SPSS Inc., Chicago, IL, USA).

\section{Results}

\section{The cocktail ACP expression of exfoliated cells in the serous effusions}

The expression of the cocktail ACP in the reactive mesothelial cells was high compared with other cancer cells. Figure 1 showed positive cocktail ACP signals with high intensity pink color in the cytoplasm of the reactive mesothelial cells. All reactive mesothelial cells in the 84 cases of the benign effusions were strong positive for the cocktail ACP staining. On the other hand, very few cancer cells were ACP positive. Only 6 out of 128 cases of the malignant effusions (4.69\%) were occasionally ACP positive with moderate intensities. Although the cancer cells were not easily differentiated from the reactive mesothelial cells by the morphology, the cocktail ACP staining successfully differentiated the above two type of the cells (Figure 2). The cocktail ACP expression levels and the expression intensities in the benign effusions was significantly higher than that of the malignant effusions $(\mathrm{P}<0.01)$. No cocktail ACP positive signals were obtained in lymphocytes or granulocytes.

\section{The expression results of the cocktail ACP in the pleural effusions, ascites, and the pericardial effusions}

As shown in Table 2, in the benign serous effusions, which including the pleural effusions, ascites, and the pericardial effusions, the specificity of the cocktail ACP positive staining were $100 \%$ for the reactive mesothelial cells. While, in the malignant serous effusions, the sensitivity of 
the cocktail ACP negative staining for the cancer cells was $95.31 \%$. Although the ACP negative rates in the malignant pleural, ascites, and the pericardial effusions were subtle different at $96.20 \%, 95.00 \%$, and $88.89 \%$ respectively, there were no statistically significant difference among the three.

\section{The diagnostic efficiency of the cocktail ACP testing and the cytology examination in the serous effusions}

Table 3 summaries the sensitivity, the specificity and the accuracy of both the ACP testing and the cytological examination in the serous effusions which included the pleural effusions, ascites, and the pericardial effusions. The cocktail ACP positive staining in the mesothelial cells of the benign serous effusions was used for detecting the specificity, while the negative ACP staining in the cancer

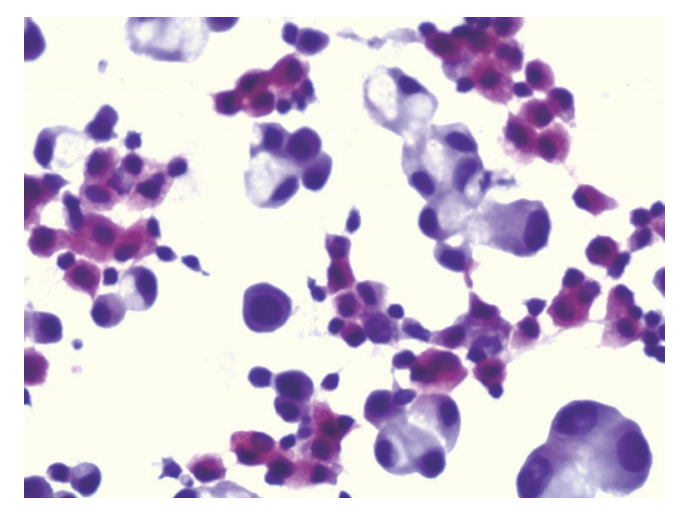

Figure 2 The cocktail ACP staining in a pleural effusion patient. The adenocarcinoma cells were arranged in an adenoid like arrangement. The adenocarcinoma cells were negative (no pink color in the cytoplasm). All the surrounding reactive mesothelial cells were positive with strong pink color in the cytoplasm. ACP staining, $\times 400$. ACP, acid phosphatases. cell cytoplasm was assigned for calculating the sensitivity. The results showed that both the sensitivity and the accuracy were improved in the ACP staining compared with the cytological examination.

\section{Discussion}

The historical data had shown that conventional cytological differential diagnosis using morphological criteria alone had a sensitivity of $69.2 \%$ and a specificity of $95.0 \%$ for detection of the malignant cells in the effusion samples (3).

Table 2 Diagnostic comparison of the cocktail ACP staining and cytology in serous fluids of patients

\begin{tabular}{cccccc}
\hline \multirow{2}{*}{ Groups } & $\mathrm{N}$ & \multicolumn{2}{c}{ Acid phosphatase } & & \multicolumn{2}{c}{ Cytology } \\
\cline { 3 - 4 } \cline { 5 - 6 } Pleural & 128 & & - & + & - \\
MC & 49 & 49 & 0 & 0 & 49 \\
AC & 79 & 3 & $76^{*}$ & 52 & 27 \\
Ascites & 69 & & & & \\
MC & 29 & 29 & 0 & 0 & 29 \\
AC & 40 & 2 & $38^{*}$ & 26 & 14 \\
Pericardial & 15 & & & & \\
MC & 6 & 6 & 0 & 0 & 6 \\
AC & 9 & 1 & $8^{*}$ & 5 & 4 \\
Total & 212 & & & & \\
MC & 84 & 84 & 0 & 0 & 84 \\
AC & 128 & 6 & $122^{*}$ & 83 & 45 \\
\hline
\end{tabular}

${ }^{*}, \mathrm{P}<0.01$ as compared to cytology. Pleural, pleural effusion; pericardial, pericardial effusion; MC, mesothelial cells; AC, adenocarcinoma cells.

Table 3 Statistical comparison of the cocktail ACP staining with cytology in diagnosis of serous effusions (pleural effusion, ascites, and pericardial effusion) $( \pm 95 \%$ CI)

\begin{tabular}{lccc}
\hline Variable & Sensitivity & Specificity & Accuracy \\
\hline Serous effusions & $95.31( \pm 3.66)$ & $100( \pm 0.00)$ & $97.17( \pm 2.23)$ \\
Pleural effusion & $96.20( \pm 4.21)$ & $100( \pm 0.00)$ & $97.66( \pm 2.62)$ \\
Ascites & $95.00( \pm 6.75)$ & $100( \pm 0.00)$ & $97.10( \pm 3.96)$ \\
Pericardial effusion & $88.89( \pm 20.53)$ & $100( \pm 0.00)$ & $93.33( \pm 12.62)$ \\
Cytology & $64.84( \pm 8.27)$ & $100( \pm 0.00)$ & $78.77( \pm 5.50)$ \\
\hline
\end{tabular}

*, $\mathrm{P}<0.01$ compared with cytology. $\mathrm{Cl}$, confidence intervals; $\mathrm{ACP}$, acid phosphatases. 
Thus, increasing the diagnostic rate which included both the specificity and the sensitivity of the malignant cells in the body fluid effusions will have an impactful effect for cancer therapy. To make the progress, the key step is to find a useful marker(s) to differentiate the cancer cells from the reactive mesothelial cells. Because the above two cell types are similar in morphology, thus cytological diagnosis of the body fluid effusion based on morphology alone is a diagnostic challenge. Especially when the number of the cancer cells is few or atypia is mild or minimal (2). False negative results are more common than false positive, and which results in the problematic effusions diagnoses $(8,18)$.

Over the years, ICC tumor markers were used to improve the sensitivity and the specificity of distinguishing the carcinoma cells from the reactive mesothelial cells in the body fluid effusion. Most of the markers were specific markers for the adenocarcinoma cells. The cocktail ACP reagent used in our current study was purchased from JCK Biopharmaceuticals, Hefei, China. It contained 5 kinds of ACPs and the main component is ACP5. Inspired by a report that the ACP was used as a tumor marker for the screening of cervical intraepithelial lesions on Pap smears (13), we originally collaborated with the vendor to test the reagent in the cervical cancer patients. Incidentally in our lab, we found that the ACP expression levels were much higher in the reactive mesothelial cells when compared to the lung adenocarcinoma cells. Based on this discovery and with modified protocol, we successfully developed the cocktail ACP as a tumor marker which had exceedingly high expression for the reactive mesothelial cells and almost no expression in the cancer cells. The cocktail ACP is not only suitable for detecting inflammation and tuberculous effusions, but also for detecting other type of the benign effusions that containing the reactive mesothelial cells. With high specificity (100\%) and high sensitivity (95.31\%), our results indicated that this staining could be an important tool in conjunction with cytomorphological diagnosis and be superior to the common immunocytochemical markers in identifying the reactive mesothelial cells including HBME-1 and calretinin (19,20). The ACP cocktail staining differentiated the reactive mesothelial cells from the adenocarcinoma cells after only 5 minutes. The positive signals were detected by pink color in the cytoplasm of the mesothelial cells. The adenocarcinoma cells were negative (no pink color) in the cytoplasm within 5 minutes (Figure 2). In fact, both the mesothelial cells and the adenocarcinoma cells had ACPs expression, but the expression levels were not the same. The mesothelial cells had higher expression levels compared with that of the adenocarcinoma cells. Thus, the key step in the cocktail ACP staining was that the ACP staining time must be restricted to 5 minutes. Within 5 minutes, the cocktail ACP reached the saturated levels in the reactive mesothelial cells. After 5 minutes the cocktail ACP levels started to reach saturation in the cancer cells. Using conventional cytological smears for the cocktail ACP detection also had other advantages, such as they were cheaper compared with immunohistochemistry (IHC), simple procedure, and quick results. They do not need paraffin embedding and special expensive equipment.

In conclusion, although the morphologies between the reactive mesothelial cells and the cancer cells were similar, the cocktail ACP expression levels were dramatically different. By applying the cocktail ACP staining as a tumour biomarker, we could successfully differentiate the benign from the malignant effusions. Thus, the cocktail ACP staining can be used as an impactful tool to complement cytological diagnosis and provide a better guidance for the clinical decision making.

\section{Acknowledgements}

Funding: This work was supported by grants from the National Natural Science Foundation of China to GuangPing Wu (grant No.81171650 and 81672082).

\section{Footnote}

Conflicts of Interest: The authors have no conflicts of interest to declare.

Ethical Statement: The study was approved by institutional ethics board of the First Affiliated Hospital of China Medical University (No. 2016-125). We have obtained internal board approval and/or patients informed consent for this study.

\section{References}

1. Figlin R, Mendoza E, Piantadosi S, et al. Intrapleural chemotherapy without pleurodesis for malignant pleural effusions. LCSG Trial 861. Chest 1994;106:363S-366S.

2. Shi YQ, He Q, Zhao YJ, et al. Lectin microarrays differentiate carcinoma cells from reactive mesothelial cells in pleural effusions. Cytotechnology 2013;65:355-62.

3. Matreja SS, Malukani K, Nandedkar SS, et al. Comparison of efficacy of cell block versus conventional smear study in 
exudative fluids. Niger Postgrad Med J 2017;24:245-9.

4. Mohanty SK, Dey P. Serous effusions: diagnosis of malignancy beyond cytomorphology. An analytic review. Postgrad Med J 2003;79:569-74.

5. El Hag M, Schmidt L, Roh M, et al. Utility of TTF-1 and Napsin-A in the work-up of malignant effusions. Diagn Cytopathol 2016;44:299-304.

6. Davidson B, Stavnes HT, Hellesylt E, et al. MMP-7 is a highly specific negative marker for benign and malignant mesothelial cells in serous effusions. Hum Pathol 2016;47:104-8.

7. Zhai K, Wang W, Wang Y, et al. Diagnostic accuracy of tumor markers for malignant pleural effusion: a derivation and validation study. J Thorac Dis 2017;9:5220-9.

8. Afify AM, Stern R, Michael CW. Differentiation of mesothelioma from adenocarcinoma in serous effusions: the role of hyaluronic acid and CD44 localization. Diagn Cytopathol 2005;32:145-50.

9. Moss DW, Raymond FD, Wile DB. Clinical and biological aspects of acid phosphatase. Crit Rev Clin Lab Sci 1995;32:431-67.

10. Yousef GM, Diamandis M, Jung K, et al. Molecular cloning of a novel human acid phosphatase gene (ACPT) that is highly expressed in the testis. Genomics 2001;74:385-95.

11. Muniyan S, Chaturvedi NK, Dwyer JG, et al. Human prostatic acid phosphatase: structure, function and regulation. Int J Mol Sci 2013;14:10438-64.

12. Goto T, Kawano H, Akiyama T, et al. Serum acid phosphatase can be a useful tumour marker for giant cell tumour of bone. Arch Orthop Trauma Surg

Cite this article as: Li YH, Wang Y, Chen XX, Liang B, Qiu XS, Wang EH, Wu GP. Diagnostic value of acid phosphatases (ACP) in differentiating reactive mesothelial cells from cancer cells in the body fluid effusions. J Thorac Dis 2018;10(12):64466451. doi: $10.21037 / j$ td.2018.11.64
2009;129:1641-4.

13. Markovic O, Markovic N. Cervical acid phosphatase: a biomarker of cervical dysplasia and a potential surrogate endpoint for colposcopy. Dis Markers 2003;19:279-86.

14. Tsuburaya RS, Monma K, Oya Y, et al. Acid phosphatasepositive globular inclusions is a good diagnostic marker for two patients with adult-onset Pompe disease lacking disease specific pathology. Neuromuscul Disord 2012;22:389-93.

15. Janckila AJ, Slone SP, Lear SC, et al. Tartrate-resistant acid phosphatase as an immunohistochemical marker for inflammatory macrophages. Am J Clin Pathol 2007;127:556-66.

16. Bull H, Murray PG, Thomas D, et al. Acid phosphatases. Mol Pathol 2002;5 5:65-72.

17. Kirschenbaum A, Liu XH, Yao S, et al. Prostatic acid phosphatase is expressed in human prostate cancer bone metastases and promotes osteoblast differentiation. Ann N Y Acad Sci 2011;1237:64-70.

18. Politi E, Kandaraki C, Apostolopoulou C, et al. Immunocytochemical panel for distinguishing between carcinoma and reactive mesothelial cells in body cavity fluids. Diagn Cytopathol 2005;32:151-5.

19. Wu GP, Zhang SS, Fang CQ, et al. Immunocytochemical panel for distinguishing carcinoma cells from reactive mesothelial cells in pleural effusions. Cytopathology 2008;19:212-7.

20. Lv M, Leng JH, Hao YY, et al. Expression and significance of MOC-31 and calretinin in pleural fluid of patients with lung cancer. Diagn Cytopathol 2015;43:527-31. 